Conclusions We identified notable differences in microbial diversity and GM composition in PD patients compared to HCs that, along with nutritional data, enabled the development of predictive modelling to identify PD. These findings further support the GM as a potentially useful biomarker of PD pathophysiology.

\section{GENETIC EPIDEMIOLOGY OF MOTOR NEURON DISEASE: ROYAL BRISBANE AND WOMEN'S HOSPITAL COHORT}

Po Sheng Yang, Robert Henderson, Pamela McCombe. Neurology, Royal Brisbane and Women's Hospital, Brisbane, QLD, Australia

\subsection{6/bmjno-2021-ANZAN.16}

Introduction Motor neuron disease (MND) can be sporadic or familial, with approximately $10 \%$ of patients being familial. A number of genetic variants have been found in patients with or without family history. We present the results of genetic testing of a cohort of MND patients diagnosed at Royal Brisbane and Women's Hospital (RBWH).

Methods The criteria for genetic testing for our cohort were consenting MND patients with or without family history. Patients were tested for C9ORF72 repeat expansions and also with a panel of MND genes. The frequency of genetic variants and their clinical features were analysed and compared.

Results There were 47 patients with a genetic variant. The most common variants were in C9ORF72 (27 patients; 57\%), SOD1 (12 patients; 26\%) and TARDBP (4 patients; 9\%). Other genes with variants were TBK1, SPAST, NEK1 and HSPB1 (one patient for each gene). The median age of onset was 50, 49 and 58 years for C9ORF72, SOD1 and TARDBP, respectively. Survival was 813,850 and 1889 days for C9ORF72, SOD1 and TARDBP, respectively. The frequency of bulbar onset was $26 \% ; 8 \%$ and $50 \%$ for C9ORF72, SOD1 and TARDBP, respectively. A family history of MND was present in $63 \%, 83 \%$ and $50 \%$ of C9ORF72, SOD1 and TARDP patients, respectively.

Conclusion The frequencies of genetic variants in our cohort are similar to what is described in European populations. Identification of genetic variants will aid the understanding of the associated phenotypes and identifying patients for possible therapy.

\section{NEUROPHOBIA: A STUDY OF AUSTRALIAN MEDICAL STUDENTS AND JUNIOR DOCTORS}

${ }^{1}$ Alex Yeung, ' 2 eila Karimi, 'Tissa Wijeratne. 'Western Health, Melbourne, VIC, Australia; ${ }^{2}$ La Trobe University, Melbourne, VIC, Australia

\subsection{6/bmino-2021-ANZAN.17}

Objectives Neurological disorders are the leading cause disability in Australia and the world. Combating the perceived difficulty of neurology or 'neurophobia' and improving physician education is a key component in addressing this problem. We aim to conduct the first study to identify whether neurophobia exists in medical students and junior doctors in an Australian population and try to identify factors that may contribute to this in this population.
Method A 24 question online validated survey was distributed via email broadcast to all medical students and junior doctors at a metropolitan tertiary care centre in Australia. Responses were collected over 6 weeks with weekly reminder emails for 4 weeks after the initial invitation email.

Results 114 medical students and junior doctors participated in the study. Participants perceived neurology as the most difficult medical speciality compared to 10 other medical specialties $(p=0.001)$. The top three factors contributing to this perceived difficulty were: a lack of understanding of neuroanatomy, lack of diagnostic certainty and lack of clinical exposure. $65 \%$ of the participants stated that they had too little planned teaching in neurology with only $36 \%$ of the participants having performed a neurology rotation during medical school.

Conclusion The prevalence of neurophobia in this Australian cohort of medical students and junior doctors is consistent with previous findings from around the world. This concerning finding requires further examination into the contributing factors in order to created trials of targeted interventions in order to resolve this.

\section{OPTIMISING SELECTION FOR EPILEPSY SURGERY}

${ }^{1,2}$ Anthony Khoo, ${ }^{1,2}$ Jane de Tisi, 'Shahidul Mannan, ${ }^{3}$ Aidan O'Keeffe, ${ }^{1,2}$ Josemir W Sander, ${ }^{1,2}$ John S Duncan. 'National Hospital for Neurology and Neurosurgery, Queen Square, London, UK; ${ }^{2}$ Department of Clinical and Experimental Epilepsy, UCL Queen Square Institute of Neurology, London, UK; ${ }^{3}$ Department of Statistical Science, UCL, London, UK

\subsection{6/bmjno-2021-ANZAN.18}

Objective To determine reasons for adult patients with drugresistant focal epilepsy who undergo presurgical evaluation not proceeding with surgery and identify factors that influence this decision.

Methods We analyzed demographic, imaging and electroclinical data on 617 consecutive patients brought to the Queen Square presurgical epilepsy MDT between January 2015 and December 2019. Multivariable logistic regression was performed to identify predictors of not proceeding with surgery, using comparative data from a prospectively-followed cohort of individuals who had epilepsy surgery at the same centre over an identical 5-year period.

Results A definitive decision not to proceed with surgery was made in 315 (51\%) cases. Common reasons behind this were an inability to localise the epileptogenic zone $(n=104)$, multifocal epilepsy $(n=74)$ and patients' decisions not to proceed with intracranial EEG $(n=50)$ or surgery $(n=39)$. Learning disability (OR: 2.35; 95\% CI 1.07-5.16), normal MRI (OR: 6.68; 95\% CI 3.71-12.05), extratemporal epilepsy (OR: 2.93; $95 \%$ CI 1.82-4.71) and bilateral seizure onset zones (OR 3.05 ; 95\% CI 1.41-6.61) were independent predictors of not having surgery. Probability of having surgery in those with normal MRI and extratemporal epilepsy was $<10 \%$. Those who did not proceed to surgery resided in more deprived socio-economic areas (median deprivation decile $40-50 \%$ vs 50-60\%, p <0.05).

Conclusions Although underutilized, epilepsy surgery is only appropriate for selected individuals with drug-resistant focal epilepsy. A predictive model based on demographic, imaging and electroclinical data can help determine those unlikely to be suitable for surgery and aid the decision to refer for more extensive or invasive evaluation. 